\title{
Über den Einfluss des Novasurols auf den Durchgang des Kongorots durch uranvergiftete Nierenepithelien.
}

\author{
(Studien über Flüssigkeitsaustausch. XXXIX. Mitteilung.) \\ Von
}

Minoru Terui.

(照 苏 實)

(Aus der Medizinischen Klinik von Prof. Dr. T. Kato, Tohoku Reichsuniversität zu Sendai.)

Früher hat Yamaguchi ${ }^{1)}$ die Untersuchung über die Permeabilität der Nierenepithelien für Farbstoffe an isolierten überlebenden Krötennieren angestellt. Bei diesem Experiment ging er so vor, dass er, indem er die Niere mit normaler Ringerlösung von der Nierenarterie sowie von der Nierenpfortader aus durchspülte, eine $0,1 \%$ ige KongorotRingerlösung von den Ureteren aus nach dem Tubuluslumen hin retrograd einströmen liess und dann die Kongorotmenge, welche in der $V$. cava post. erschien, durch Bestimmungen beobachtete. Bei diesem Verfahren konnte bei der gesunden Niere das Kongorot nicht durch die Tubulusepithelien passieren, so dass die Durchspülungsflüssigkeit aus V. cava post. sich gar nicht oder nur in Spuren färbte und zwar in so geringen Mengen, die zu quantitativen Bestimmungen nicht gelangen konnten. Wenn man aber die Tubulusepithelien durch Vorbehandlung mit Sublimat deren Funktion herabgesetzt hatte, vermochte die TubuIuswand dem Durchgang von Kongorot nicht entgegenzuwirken, so dass dieser Farbstoff in der Durchströmungsflüssigkeit aus V. cava post. deutlich zum Vorschein kam.

Im vorliegenden Versuch wurde die Beobachtung darüber angestellt, was für einen Einfluss das Novasurol auf den Durchgang des Kongorots durch die Tubulusepithelien, deren Funktion durch Uranylnitrat zuvor beeinträchtigt worden ist, ausüben würde.

Die Wirkung von Novasurol auf die Tubulusepithelien ist durch

1) Xamaguchi, Takahashi, Takeda L. Shoji, Tohoku Journ. Exp. Med., $1933,20,331$. 
Untersuchung von Tada $\mathrm{u}$. Saito $\mathrm{o}^{27}$ und Izu mida $\mathrm{a}^{3)}$ klargestellt worden; das Novasurol soll die Funktion der Tubulusepithelien, also rïckresorptionshemmendes Vermögen steigern. Shoji ${ }^{4}$ ) beobachtete an uranvergifteter Krötenniere, dass das intratubulär eingeführte Kreatinin in seinem Durchgang durch die Tubuluswand durch Novasurolwirkung gehindert wurde.

Versuchsmethode: Die Präparate der Krötenniere wurden, nach Ablauf von $24 \sim 48$ Std., nachdem man 0,5 ccm einer $2 \%$ igen Uranylnitratlösung pro $100 \mathrm{~g}$ Körpergewicht in den Hinterschenkel der Kröte injiziert hatte, nach dem Verfahren, wie es in IX. ${ }^{2)}$ Mitteilung einschlägiger Studien ausführlich beschrieben ist, hergestellt. DasKongorot wurde im Verhältnis von 0,1\% in Ringerlösung gelöst verwendet. Die Durchspülung wurde nach der Methode, wie sie in XI..5) Mitteilung einschlägiger Studien ausführlich beschrieben wurde, durchgeführt; die Ausfuhrmenge aus $V$. cava post. wurde alle 5 min angesammelt, die darin vorhandene Kongorotmenge wurde mit Hilfe des Colorimeters von Duboscq gemessen und weiterhin wurde an einzelnen Portionen das prozentische Verhältnis der Kongorotmenge in der Ausfuhr zu der in das Tubuluslumen eingeströmten Kongorotmenge errechnet, damit der zeitliche Verlauf von Kongorotausscheidung beobachtet werden konnte.

\section{Kontrollversuch. (Fig. 1)}

In Kontrollversuch wurde das Kongorot in die Uranniere, ohne dass man zuvor das Novasurol auf dieselb einwirken liess, eingeführt, und die Ausfuhr wurde ermittelt.

Hierbei wurden 6 Versuche angestellt. Indem man eine $0,1 \%$ ige Kongorot-Ringerlösung fortlaufend von den Ureteren aus intratubulär einströmen liess, hat man die Krötenniere mit normaler Rịngerlösung von der Nierenarterie oder von der Nierenpfortader aus durchspült. Der Prozentsatz der Kongorotmenge in der Ausfuhr zu der in der $\mathrm{Zu}-$ fuhr zeigte bei allen Versuchen, mitunter unter mässigen Schwankungen, die Tendenz, mit demZeitablauf allmählich immer höher anzusteigen. Es gab aber manche Versuche, in denen nach Ablauf von $60 \sim 70$ min eine Hinneigung zum Absinken angetroffen wurde.

Die von der Ureteren aus intratubulär eingeführte Kongorot-

2) Tad a u. S a i to (IX. Mitteilung), Tohokx Journ. Exp. Med., 1930, 15, 91.

3) I z u mid a, Ibid, 1939, 36, 63.

4) S h o ji, Ibid., 1936, 29, 8.

5) XII. Mitteilung (Y a m a g u chi), Ibid., 1932, 18, 392. 
Ringerlösung nahm mit der Zeit allmählich im geringen Grad an Mengen zu. Die Zufuhrmenge von der Nierenarterie u. -pfortader aus nahm hingegen allmählich ab.

Fig. 1. Kontrollversuch (Versuch 5).

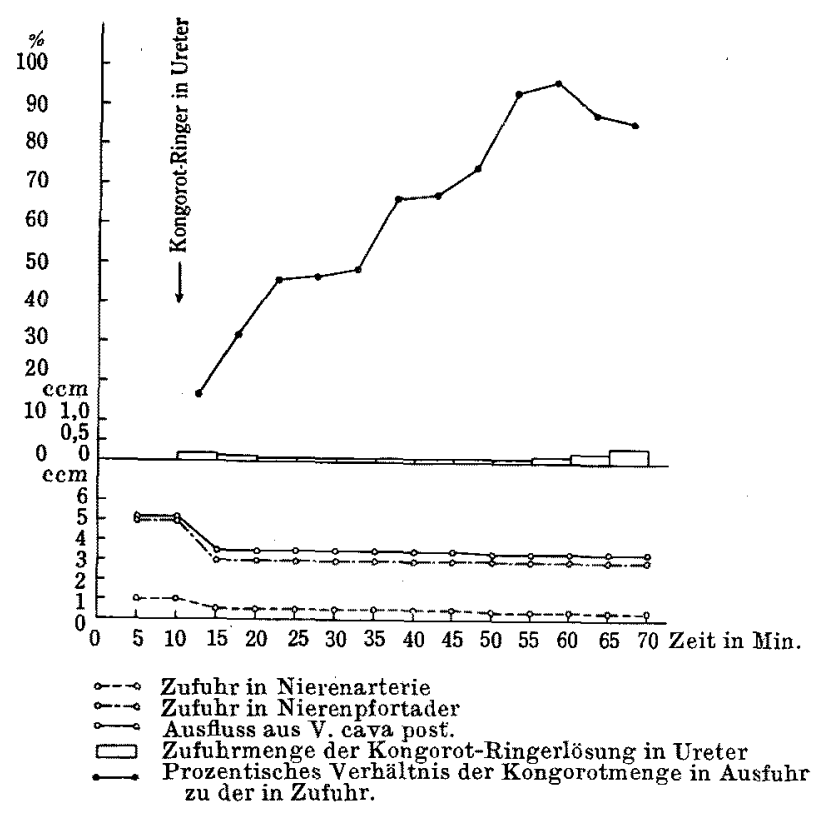

Die Tatsache, dass die von den Ureteren eingeströmte Flüssigkeitsmenge sowie der Prozentsatz der Kongorotmenge in der Ausfuhr zu der in der Zufuhr, wie oben erwähnt immer mehr zunehmen, dürfte wahrscheinlich damit in Zusammenhang gebracht werden, dass erkrankte Nierenepithelien durch Sauerstoffmangel funktionell immer intensiver erschöpft sind, wodurch ihre rückresorptionsregulierende Fähigkeit allmählich beeinträchtigt worden ist.

\section{Zufuhr des Novasurols von der Nierenpfortaderaus. (Fig. 2)}

Es wurden hier 5 Versuche angestellt. Nachdem zuerst die Durchspülung nach demselben Verfahren wie im Kontrollversuch vorgenommen worden war, wurde 30 min später die Normal-Ringerlösung von Nierenpfortader aus auf eine $0,1 \%$ ige Novasulol-Ringerlösung umgeschaltet. Man liess nämlich das Novasurol von der Rückenseite der Tubuli her einwirken. Bei diesem Verfahren erfuhr der Prozentsatz von Kongorot, der sich auf dem Wege der Erhöhung befand, unter dem 
Fig. 2. Novasurolzufuhr von Nierenpfortader aus (Versuch 1).

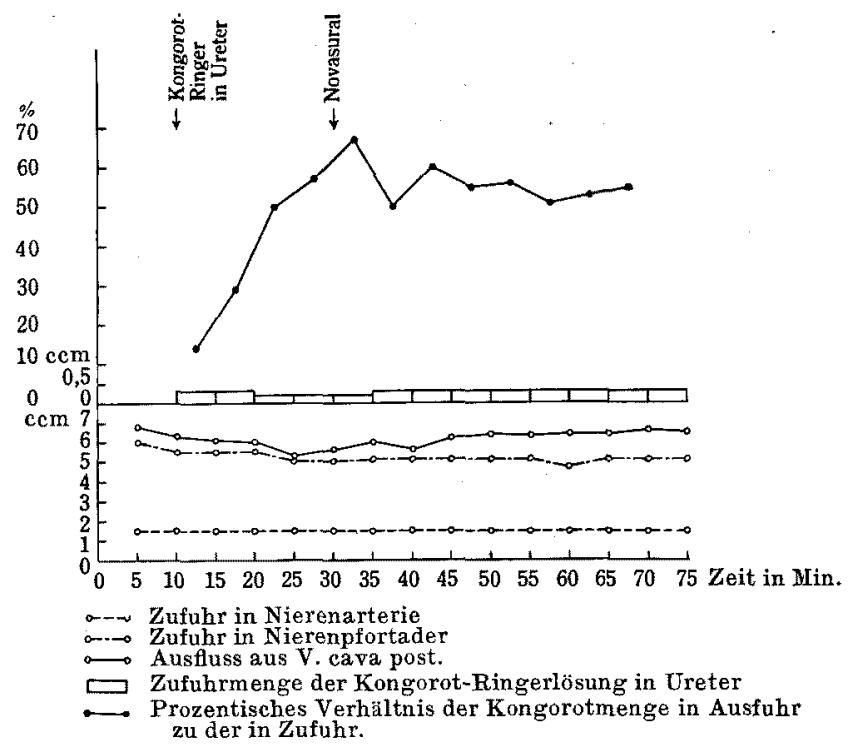

Einfluss der Novasurolwirkung entweder eine allmähliche Abnahme oder nahm, unter geringen Schwankungen, einen annähernd stationären Verlauf. In Zeitabschnitten aber, in denen die Novasurol-Ringerlösung wieder auf Normal-Ringer umgeschaltet wurde, stieg der Prozentsatz von neuem an. Die Veränderungen der intratubulär eingeführten Mengen von Kongorot-Ringerlösung sowie der Zufuhrmenge von der Nierenarterie $u$. -pfortader aus verhielten sich in beinahe analoger Weise, wie im Kontrollversuch. Mitunter gab es aber Versuche, in denen die Zufuhrmenge von der Nierenpfortader aus mitten auf der Versuchsdauer zuzunehmen anfing.

\section{Intratubuläre Novasurolzufuhr von den Ureteren aus. (Fig. 3)}

Es wurden 5 Versuche ausgeführt.

Am Anfang wurde in gleicher Weise wie im Kontrollversuch vorgegangen. $30 \mathrm{~min}$ später liess man eine $0,1 \%$ ige Novasurol-KongorotRingerlösung anstatt der Kongorot-Ringerlösung von den Ureteren aus einfliessen. Man liess nämlich das Novasurol von der Lumenseite her direkt auf die Tubulusepithelien einwirken. Bei diesem Verfahren nahm der Prozentsatz von Kongorot, der sich zu weiterer Erhöhung anschickte, durch Novasurolwirkung unter etlichen Schwankungen 
Fig. 3. Novasurolzufubr in Ureter (Versuch 2).

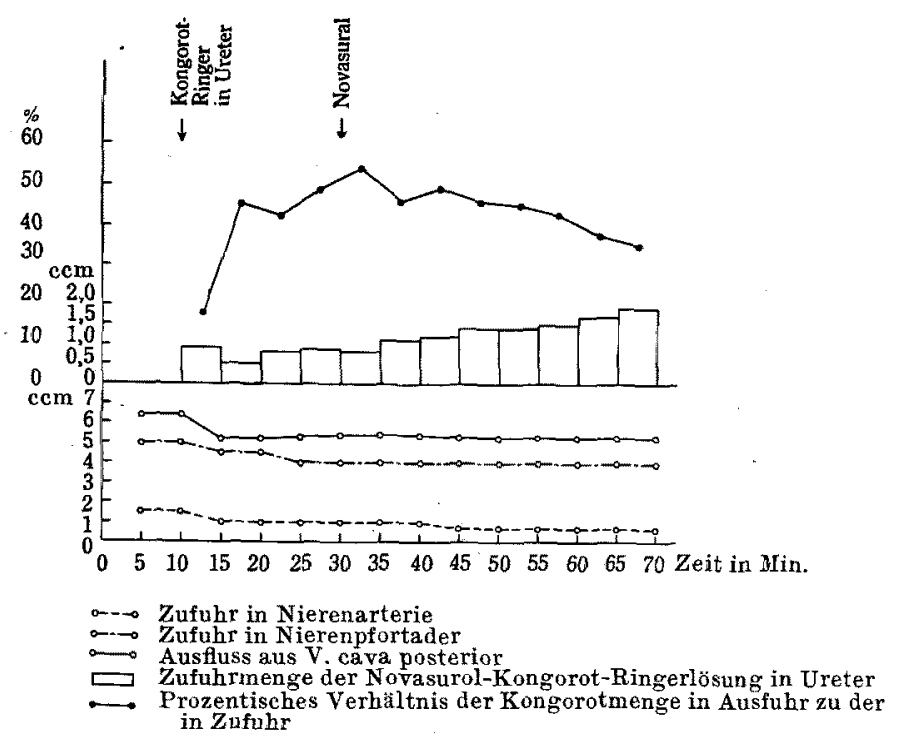

einen absinkenden Verlauf. Die in das Tubuluslumen eingeflossene Flüssigkeitsmenge sowie die Zufuhrmenge von der Nierenarterie u. -pfortader aus verhielten sich fast analog wie im Kontrollversuch.

Nach oben erwähnten Resultaten dürfte das Novasurol bei der Uranniere, deren Tubuluswand für das Kongorot durchlässig geworden war, auf diese Durchlässigkeit hemmend wirken.

Nach der Yamaguchis ${ }^{6}$ Ansicht lässt sich folgenderweise erklären, dass dem Novasurol die Fähigkeit zukommt, die Tubulusepithelien zu reizen und deren rückresorptionsbeschränkendes Vermögen zur Steigerung zu bringen, wodurch die Tubulusepithelien in den Stand gesetzt werden, dem Durchgang von Kongorot durch die Tubuluswand stärker entgegenzuwirken. Die Tatsache indessen, dass die ins Tubuluslumen eingeflossene, durch die Kanälchenwand hindurch gegangene Menge von Kongorot-Ríngerlösung durch Novasurol quantitativ kaum beeinflusst wird, dürfte dahin gedeutet werden, dass die rückresorptionsbeschränkende Kraft der Tubulusepithelien weniger auf kleinmolekuläre Substanzen, als vielmehr auf grossmolekuläre Substanzen wirkt, d.h. dass, trotzdem die Rückresorption des Kongorotes gehemmt wird, die Ruickresorption des Wassers hingegen nicht hintangehalten wird.

6) Y a magu chi, Tohokn Journ. Exp. Med., 1930, 16, 361. 


\section{Schluss.}

An isolierten überlebenden, uranvergifteten Krötennieren wurde der Einfluss des Novasurols auf den Durchgang von Kongorot durch die Tubuluswand untersucht, woraus sich folgende Schlussfolgerung ergab:

Das Novasurol wirkt dem Durchgang von Kongorot durch die Tubuluswand in der Weise entgegen, indem das Novasurol die den Tubulusepithelien zukommende Funktion, also die regulierende bzw. beschränkende Kraft auf die Rückresorption verstärkt.

Zum Schluss spreche ich vom ganzen Herzen meinen aufrichtigen Dank aus Herrn Dr. Yamaguchi, dem vor kurzem dahingeschiedenen Privatdozenten für seinen liebenswürdigen eifrigen Beistand bei meiner Arbeit. 